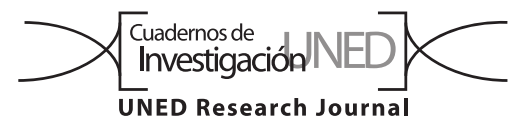

NOTA

\title{
Creencias en salud en una población militar joven, en relación con el tabaquismo
}

\author{
Laura Elizabeth Castro', ${ }^{1}$, Ana Isabel Garcia Muñoz ${ }^{1,2}$, Edson Camilo Lozada Gutierrez ${ }^{1,2}$, \\ Jenner Rodrigo Cubides Amezquita ${ }^{1,2}$ \& Laura Tatiana Calderon Molina ${ }^{1,2}$ \\ 1. Escuela Militar de Cadetes General José María Cordova, Colombia; lauraunica12@gmail.com \\ 2. Universidad Santo Tomás, Colombia.
}

Recibido 20-VIII-2018 • Corregido 01-X-2018 • Aceptado 13-XI-2018

\begin{abstract}
Beliefs in health in a young military population, in relation to smoking". In the military population, tobacco consumption is associated with age, stress, family separation and team pressure. We applied a survey to 29 Colombian cadets whose average age was 22 years. They associate smoking with self-confidence, insomnia, better digestion and pleasure; as well as with cancer, coughing, throat irritation, lung diseases and brain damage. Nevertheles, $42 \%$ have smoked, and consume 1 to 5 cigarettes daily.
\end{abstract}

Keywords: health promotion, tobacco use disorder, health knowledge, attitudes, practice.
RESUMEN: En la población militar, el consumo de tabaco está asociado con la edad, el estrés, la separación familiar y la presión de grupo. Aplicamos una encuesta a 29 cadetes colombianos cuya edad promedio era de 22 años. Asocian fumar con autoconfianza, insomnio, mejor digestión y placer; así como con cáncer, tos, irritación de garganta, enfermedades pulmonares y daño cerebral. Sin embargo, el $42 \%$ ha fumado y consume de 1 a 5 cigarrillos al día.

Palabras Clave: promoción de la salud, tabaquismo, conocimientos, actitudes y práctica en salud.
El tabaquismo es uno de los mayores problemas de salud pública (Gutiérrez, 2003), ya que es un factor de riesgo cardiovascular y cardiorespiratorio, siendo asociado a la presencia de la mayoría de las enfermedades crónicas no transmisibles. Reportes de la Organización Mundial de la Salud han informado que hay 1100 millones de fumadores a nivel mundial, un tercio de la población mayor de 15 años aproximadamente. Hay un 47\% de hábito de fumar para hombres y un $11 \%$ mujeres, con un consumo medio de 14 cigarrillos/día, lo que supone un total de 5827 billones de cigarrillos al año (Gutiérrez, 2003). Por ello es prioritario disminuir el consumo (Forés et al., 2017).

En la población militar joven hay una alta incidencia de este hábito, por factores como la edad, la separación circunstancial con sus familias y la presión de grupo (Ortiz, Díaz, Gamba, Ibáñez, \& Hernández, 2013). Para atender el problema, deben conocerse las creencias de la población sobre ventajas y desventajas de fumar (Arrivillaga, Salazar, \& Correa, 2003; Lara, Yáñez, Guzmán, \& Salomón, 2014). Acá informamos sobre las creencias en salud del tabaquismo en estudiantes de una escuela militar colombiana.

Usamos el "Cuestionario de creencias sobre los efectos del tabaco (CCET)”, de García-García, Vázquez-Galindo, Hayashida y Santos (2014), adaptado al lenguaje local. El intrumento tiene tres partes, una sobre datos de consumo, la segunda parte sobre los beneficios de fumar y la tercera los contras de fumar, utilizando una escala de Likert con cuatro opciones de respuesta. Añadimos datos sociodemográficos y sobre tiempo libre, aplicando el instrumento a 29 cadetes de séptimo nivel que firmaron consentimiento informado.

El $90 \%$ son hombres, la media de edad es de $21,72 \mathrm{DE} \pm$ 1,38 . Un $42 \%$ ha fumado, de estos el $4 \%$ inició a los 12 años, seguido del grupo de 15 a 19 años. El 37\% ha fumado en el último año, el $30 \%$ en el último mes y $30 \%$ en la última semana. Consumen de 1 a 5 cigarrillos diarios. Afirmaron que fumar les da seguridad en si mismos (71,5\%), quita el sueño (65\%), ayuda a la digestión (75\%) y brinda sensaciones de placer (72\%). 
Respondieron que el tabaco provoca cáncer $(66,7 \%)$, tos $(81,4 \%)$, irritación de garganta $(85,1 \%)$, genera enfermedades pulmonares $(92,5 \%)$ y disminuye la actividad de las neuronas $(85,1 \%)$. Se hizó un análisis bivariado entre fumar y las creencias que se tienen en tabaquismo (Apéndice 1).

El hábito tabáquico se ha asociado, entre otros, a las creencias que se construyen, reconfiguran y transmiten de generación en generación. En nuestro estudio se evidencia una mayor percepción en contra del tabaco, particularmente sobre la adicción, lo que puede favorecer su abandono (García-García et al., 2014). En el presente estudio, las creencias negativas más referidas, fueron las relacionadas con daños a la salud, lo que concuerda con el trabajo de Gupta y Kumar (2014), realizado en 14 países, en el cual se evidenció que estos temores, en edades entre 15 a 24 años, se asocian a menor deseo de consumir. De hecho, se ha documentado que el temor a desenlaces crónico-degenerativos a largo plazo conduce a menor probabilidad de adquirir el hábito y por ello se ha recomendado utilizar dicha preocupación, en pro de su no consumo, en las intervenciones que se implementen (Wellman et al., 2018).

La tos y pérdida de condición física también se da en soldados egipcios, haciéndolos querer dejar el hábito (Khattab, AbdelFattah, \& Abozahra, 2017). Aunque en este estudio, las creencias sobre los beneficios del tabaquismo fueron menos respaldadas, igualmente son preocupantes, como la creencia de que fumar hace rico y placentero el momento, principal motivo para iniciar el consumo (Mbatchou-Ngahane, Luma, Mapoure, Fotso, \& Afane, 2013). Al igual que en el etudio de Baig et al., (2016), fumar, para los jóvenes, es un signo de madurez y los ayuda a incorporarse socialmente.

Esta población sigue fumando a pesar de reconocer las consecuencias negativas del consumo de tabaco, por lo que es necesario estudiar porqué lo hacen.
Ética, conflicto de intereses y declaración de financiamiento: los autores declaran haber cumplido con todos los requisitos éticos y legales pertinentes, tanto durante el estudio como en el manuscrito; que no hay conflictos de interés de ningún tipo, y que todas las fuentes financieras se detallan plena y claramente en la sección de agradecimientos. Asimismo, están de acuerdo con la versión editada final del documento. El respectivo documento legal firmado se encuentra en los archivos de la revista.

\section{AGRADECIMIENTOS}

Los autores agradecen a los participantes del estudio y al director de la Escuela Militar de Cadetes "General José María Córdova" donde se realizó el estudio.

\section{REFERENCIAS}

Arrivillaga, M., Salazar, I.C., \& Correa, D. (2003). Creencias sobre la salud y su relación con las prácticas de riesgo o de protección en jóvenes universitarios. Colombia Médica, 34(4), 186-195.

Baig, M., Bakarman, M.A., Gazzaz, Z.J., Khabaz, M.N., Ahmed, T.J., Qureshi, I.A., ... Altherwi, F.B. (2016). Reasons and Motivations for Cigarette Smoking and Barriers against Quitting Among a Sample of Young People in Jeddah, Saudi Arabia. Asian Pacific Journal of Cancer Prevention, 17(7), 3483-3487.

Forés, R., Alzamora, M.T., Pera, G., Valverde, M., Angla, M., BaenaDíez, J.M., \& Mundet-Tuduri, X. (2017). Evolución y grado de control de los factores de riesgo cardiovascular tras 5 años de seguimiento y su relación con la incidencia de arteriopatía periférica: cohorte poblacional ARTPER. Medicina Clínica, 148(3), 107-113. DOI: 10.1016/j. medcli.2016.09.043 


\section{APÉNDICE 1}

Análisis bi-variado de creencias sobre los beneficios del tabaquismo en cadetes colombianos

\begin{tabular}{|c|c|c|c|c|}
\hline Variables & & $\mathrm{Si}$ & No Valor de $\mathrm{P}$ & \\
\hline \multirow[t]{5}{*}{ Hace que la gente se sienta más adulta y segura } & & $\mathrm{n}$ & $\mathrm{n}$ & \\
\hline & Muy en desacuerdo & 4 & 4 & \\
\hline & En desacuerdo & 6 & 5 & \\
\hline & De acuerdo & 0 & 5 & \\
\hline & Muy de acuerdo & 1 & 1 & 0,205 \\
\hline \multirow[t]{4}{*}{ Hace que la gente se sienta más tranquila } & Muy en desacuerdo & 3 & 2 & \\
\hline & En desacuerdo & 0 & 8 & \\
\hline & De acuerdo & 7 & 3 & \\
\hline & Muy de acuerdo & 1 & 2 & 0,021 \\
\hline \multirow[t]{4}{*}{ Hace que se te quite el sueño cuando estudias } & Muy en desacuerdo & 4 & 3 & \\
\hline & En desacuerdo & 3 & 9 & \\
\hline & De acuerdo & 4 & 2 & \\
\hline & Muy de acuerdo & 0 & 1 & 0,231 \\
\hline \multirow[t]{4}{*}{ Ayuda a inspirarse para estudiar } & Muy en desacuerdo & 4 & 6 & \\
\hline & En desacuerdo & 5 & 7 & \\
\hline & De acuerdo & 2 & 1 & \\
\hline & Muy de acuerdo & 0 & 1 & 0,685 \\
\hline \multirow[t]{4}{*}{ Ayuda a una persona a distraerse de los problemas } & Muy en desacuerdo & 1 & 5 & \\
\hline & En desacuerdo & 2 & 4 & \\
\hline & De acuerdo & 6 & 5 & \\
\hline & Muy de acuerdo & 2 & 1 & 0,359 \\
\hline \multirow[t]{4}{*}{ Ayuda a la digestión después de comer } & Muy en desacuerdo & 2 & 9 & \\
\hline & En desacuerdo & 4 & 5 & \\
\hline & De acuerdo & 3 & 0 & \\
\hline & Muy de acuerdo & 2 & 1 & 0,059 \\
\hline \multirow[t]{4}{*}{ Hace que se te quite el sueño } & Muy en desacuerdo & 4 & 6 & \\
\hline & En desacuerdo & 4 & 8 & \\
\hline & De acuerdo & 3 & 0 & \\
\hline & Muy de acuerdo & 0 & 1 & 0,155 \\
\hline \multirow[t]{4}{*}{ Hace que el momento sea rico y placentero } & Muy en desacuerdo & 3 & 8 & \\
\hline & En desacuerdo & 4 & 2 & \\
\hline & De acuerdo & 1 & 2 & \\
\hline & Muy de acuerdo & 2 & 2 & 0,444 \\
\hline \multirow[t]{5}{*}{ Hace que la gente se vuelva adicta } & & $\mathrm{n}$ & $\mathrm{n}$ & \\
\hline & Muy en desacuerdo & 2 & 1 & \\
\hline & En desacuerdo & 2 & 4 & \\
\hline & De acuerdo & 3 & 3 & \\
\hline & Muy de acuerdo & 3 & 7 & 0,644 \\
\hline \multirow[t]{4}{*}{ Provoca cáncer } & Muy en desacuerdo & 0 & 0 & \\
\hline & En desacuerdo & 0 & 2 & \\
\hline & De acuerdo & 3 & 3 & \\
\hline & Muy de acuerdo & 7 & 10 & 0,451 \\
\hline
\end{tabular}


Apéndice 1 (Continuación)

\begin{tabular}{|c|c|c|c|c|}
\hline Variables & & $\mathrm{Si}$ & No Valor de P & \\
\hline \multirow[t]{4}{*}{ Hace que la gente pierda condición física } & Muy en desacuerdo & 0 & 2 & \\
\hline & En desacuerdo & 3 & 1 & \\
\hline & De acuerdo & 3 & 5 & \\
\hline & Muy de acuerdo & 4 & 7 & 0,326 \\
\hline \multirow[t]{4}{*}{ Te provoca tos } & Muy en desacuerdo & 1 & 1 & \\
\hline & En desacuerdo & 1 & 1 & \\
\hline & De acuerdo & 4 & 8 & \\
\hline & Muy de acuerdo & 4 & 5 & 0,927 \\
\hline \multirow[t]{4}{*}{ Te causa enfermedades pulmonares } & Muy en desacuerdo & 1 & 0 & \\
\hline & En desacuerdo & 0 & 0 & \\
\hline & De acuerdo & 5 & 7 & \\
\hline & Muy de acuerdo & 4 & 8 & 0,42 \\
\hline \multirow[t]{4}{*}{ Te afecta el corazón } & Muy en desacuerdo & 1 & 0 & \\
\hline & En desacuerdo & 0 & 2 & \\
\hline & De acuerdo & 4 & 6 & \\
\hline & Muy de acuerdo & 5 & 7 & 0,416 \\
\hline \multirow[t]{4}{*}{ Disminuye la actividad en las neuronas } & Muy en desacuerdo & 1 & 0 & \\
\hline & En desacuerdo & 1 & 1 & \\
\hline & De acuerdo & 4 & 8 & \\
\hline & Muy de acuerdo & 4 & 6 & 0,614 \\
\hline \multirow[t]{4}{*}{ Te irrita la garganta } & Muy en desacuerdo & 3 & 0 & \\
\hline & En desacuerdo & 0 & 0 & \\
\hline & De acuerdo & 4 & 8 & \\
\hline & Muy de acuerdo & 3 & 7 & 0,077 \\
\hline \multirow[t]{4}{*}{ Hace que la gente tenga un mal sabor de boca } & Muy en desacuerdo & 2 & 0 & \\
\hline & En desacuerdo & 2 & 1 & \\
\hline & De acuerdo & 2 & 5 & \\
\hline & Muy de acuerdo & 4 & 9 & 0,193 \\
\hline
\end{tabular}

